\title{
CONCLUDING REMARKS
}

\author{
ZDENEK KOPAL \\ Deparment of Astronomy, University of Matchester, England
}

In conclusion of the conference just past, may I be permitted to record a few thoughts which may also have occurred to many colleagues.

First, what would Bessel have thought if he could have been with us to hear how far the science, for which he laid the foundations in the first balf of the 19th century, has progressed; if he heard what Drs Bernacca and Dommanget had to say on the impending Project HIPPARCOS, which promises to increase the accuracy of positional measures of the stars 100 times greater than that attainable by Bessel himself and his contemporaries; if he heard of laser ranging of the Moon, and of the studies of its librations with the aid of cube-corner retro-reflectors deposited by men on the surface of the Moon for this purpose?

He would, no doubt, have been astonished by such feats beyond imagination. And yet, do these and other advances accomplished in the time which elapsed since Bessel's time mean that we are so much brighter or more intelligent than our 19th-century ancestors? By no means! For any historian of science in recent times cannot fail to note that the principal reason why the advances accomplished since the time of Bessel - and, in particular, within our lifetime - have been due to no superior intelligence or understanding, but to the support of parallel advances in human technology at our disposal which made, of course, tremendous strides in the past 150 years. The motives of its magnificent efflorescence were not to advance basic science, but to apply existing knowledge to practical ends; yet is is undeniable that astronomers were important beneficiaries of this technological spin-off (and sometimes even prime contributors to it).

During Bessel's lifetime, observational astronomy was still essentially a visual art; with photography (terrestrial) making only its first steps when Bessel died in 1846; and electronics was still far in the future. The only technology which Bessel could avail himself of in support of his work was provided by mechanically-minded contemporaries like Josef Fraunhofer, Reichenbach-Ertel, Repsold, or Rheinfelder. Yet it is remarkable how far - with their aid - Bessel was able to eliminate systematic errors in his work, to obtain results astonishing in their accuracy even today.

The precision with which Bessel was able to determine the parallax of 61 Cygni with his heliometer was already paid tribute in my introductory remarks (p. 3). Another example of what his heliometer could do was provided, by his successors Schlüter and Wichmann at Königsberg (and especially Ernst Hartwig of Strassburg and Bamberg!) in their studies of physical librations of our Moon.

Of the parameters specified by the periods and amplitudes of lunar physical librations,

* Communication presented at the International Conference on 'Astrometric Binaries', held on 13-15June, 1984 , at the Remeis-Sternwarte Bamberg, Germany, to commemorate the 200 th anniversary of the birth of Friedrich Wilhelm Bessel (1784-1846). 
of paramount importance are the ratios

$$
\alpha=\frac{C-B}{A}, \quad \beta=\frac{C-A}{B}, \quad \gamma=\frac{B-A}{C},
$$

where $A, B, C$ denote moments of the Moon about its principal axes of inertia. The value of $\beta$ can be determined, in principle, from the amplitudes of the physical librations (i.e., from the inclination of the Moon's orbit and equator to the ecliptic, and the rate of regression of the nodes of lunar orbit); and that of $\gamma$, from their observed periodicity; while the value of $\alpha$ corresponding to known $\beta$ and $\gamma$ can be evaluated from the identity

$$
\alpha-\beta+\gamma=\alpha \beta \gamma \text {. }
$$

The fact that the differences $C-A, C-B$, or $B-A$ (and, therefore, the ratios $\alpha, \beta$, or $\gamma$ ) must be different from zero is amply attested by the observed synchronism between rotation and revolution of our satellite; for if the shape of the Moon were a sphere with a spherically-symmetrical distribution of matter in its interior - the Earth's attraction would be powerless to effect synchronization. The existence of the latter cannot, to be sure, by itself specify the actual amounts by which $\alpha, \beta$, or $\gamma$ may differ from zero; this can come out only from a study of the librations performed by the distorted globe of the Moon about its centre of gravity. The arduous task of determining the values of $\beta$ and $\gamma$ from long series of heliometric observations (extending over almost one century) was carried out in 1962 at Manchester by Koziel (1967a, b), with the results disclosing that

$$
\begin{aligned}
& \alpha=0.000398 \pm 0.000008 \text { (m.e.) } \\
& \beta=0.000629 \pm 0.000001 \text { (m.e.) } \\
& \gamma=0.000231 \pm 0.000006 \text { (m.e.) }
\end{aligned}
$$

It was noted many years ago (cf., for example, Jeffreys, 1924) that the observed values of $\alpha, \beta$, and $\gamma$ are completely at variance with those to be expected if the lunar globe were in hydrostatic equilibrium under the field of force to which the Moon could have been exposed. In particular, the actual value of $\beta$ proves to be approximately 17 times as large as a hydrostatic one under the prevalent field of force; while $\alpha$ and $\gamma$ are 42 and 8 times as large, respectively.

This is true, to be sure, at the present distance of the Moon from the Earth. This distance need not have remained constant throughout the long astronomical past of our satellite; and could once have been very much less - which would have influenced the absolute values of $A, B$, and $C$. But regardless of the possibility of bringing any one of the observed values of $\alpha, \beta$, or $\gamma$ in agreement with hydrostatic theory by allowing the Moon to have acquired its form in closer proximity of the Earth, the requisite proximity does not turn out to be the same for all three. In other words, the ratios of the observed lunar values of $\alpha, \beta$, and $\gamma$ prove to be inconsistent with the existence of hydrostatic (or lithostatic) equilibrium at any distance of the Moon from the Earth. 
In order to demonstrate this, let us form the ratio

$$
f \equiv \frac{\alpha}{\beta}=\frac{B}{A} \frac{C-B}{C-A} .
$$

In hydrostatic equilibrium, this should be (cf., for example, Kopal, 1969) equal to

$$
f=\frac{m_{\oplus}+m_{\mathfrak{a}}}{4 m_{\oplus}+m_{\mathfrak{a}}},
$$

where $m_{\oplus}$ and $m_{\mathbb{a}}$ denote the masses of the Earth and the Moon, respectively; and this equation should hold good regardless of the Moon's distance or internal structure. As, moreover, the latest value of the ratio $m_{\oplus} / m_{\mathbb{\pi}}$ is equal (cf. Table 3-1 of Kopal, 1974) to $81.302 \pm 0.001$ (m.e.), the 'hydrostatic' value of $f$ as given by Equation (4) should be equal to 0.2523 ; whereas the observed value of $f$ consistent with Koziel's values of $\alpha$ and $\beta$ is equal to

$$
f=0.633 \pm 0.006 \text { (m.e.) ; }
$$

and some previous investigators (e.g., Gorynia, 1965; Habibullin, 1966) have put it even higher.

These values are so much at variance with the requirements of hydrostatic equilibrium that the discrepancy must be regarded as real. Several thoughts should be considered in this connection. On the observational side, it is true that Koziel's results are based on heliometric measurements made with instruments of apertures smaller than $17.5 \mathrm{~cm}$; and their Rayleigh limits of resolution in visible light was, therefore, not less than 0.8 . In order to obtain, from such measurements, results significant to 0 ". 1 (as quoted by heliometric observers), the mean of a great many individual settings must be made on the assumption that no systematic errors are present to impair their mean to this accuracy.

Positional astronomers have, to be sure, long been accustomed to search for the desired information inside optical diffraction patterns of their objectives; and have done so extensively when measuring, for example, stellar parallaxes. The success of such a process requires, however, a knowledge of the geometrical relation of the actual shape of the light source to that of its diffraction image (i.e., a point to a disc in the case of a star), in addition to a great many individual measures to minimize their accidental errors. In the case of selenodetic measurements the first condition cannot, unfortunately, be met; for the actual shape of lunar details on which heliometric settings are made are not known to us a priori; and neither is, therefore, the form of their diffraction image (which may, moreover, vary with the phase as a result of different illumination).

Under these conditions, many of us have been veritably holding our breath until the result of heliometric studies of lunar librations could be confirmed by independent methods. This has come to pass since the landing on the Moon of Apollo 11 in July 1969, when a cube-corner retro-reflector for laser signals was installed on the shores of the lunar Mare Tranquillitatis; followed by similar devices (of improved design) installed 
by Apollo 14 and 15, or the unmanned Lunas 17 and 21, in the proximity of their landing places. Observed echoes of laser pulses flashed on the Moon from the Earth and returned by these devices - permitting determinations of the instantaneous distance between the terrestrial transmitter and the particular cube-corner reflector on the Moon from the time-delays of the respective light-echoes with a precision of the order of one part in $10^{8}$ - have opened a new epoch in several branches of lunar studies - including that of lunar librations.

At present these laser-tracking studies are still continuing; but the first published results (cf., Bender et al., 1973; Williams et al., 1973) led to the values of

$$
\begin{aligned}
& \alpha=0.0004043 \pm 0.0000011, \\
& \beta=0.0006311 \pm 0.0000004, \\
& \gamma=0.0002268 \pm 0.0000010,
\end{aligned}
$$

virtually identical with those derived from ground-based studies of lunar librations by means of terrestrial telescopes; their mean errors are somewhat smaller, but of the same order of magnitude. The internal agreement between these two sets of the data obtained by completely different means (and subject to entirely different types of errors) is indeed excellent, and inspires full confidence in the correctness of the results. It vindicates brilliantly the patient efforts of investigators like Ernst Hartwig (1851-1923) who dedicated a major part of their lifetime to this exacting task; as well as the skill which inspired investigators like Koziel to extract the requisite information from long series of observations inherited from bygone days. The only other instance of a comparable feat in the history of science one can think of has been Chapman's success (cf., Chapman, 1918) in detecting the amplitudes of the lunar atmospheric tides from the barometric data measured in the equatorial belt of the Earth, whose individual errors were 10-100 times as large as the amplitudes sought after.

Moreover, one additional consideration may be adduced, which could explain at least partly why the achievements of Friedrich Wilhelm Bessel and of his contemporaries (among whom his friend Carl Friedrich Gauss holds an especially prominent place) were not followed up by their descendents with quite the same success. Consider Bessel as an example! His principal scientific accomplishments date to the last third of his 62-years long life - i.e., a time during which most of his successors of comparable prominence (certainly in the 20 th century) would have been reduced to the role of administrators, or to public figures having little time or opportunity to pursue the promising work of their youth on which their early fame was based.

The 'brain-pollution' - largely self-inflicted - to which humanity became a victim in our times I had an opportunity to discuss on another recent occasion (Kopal, 1984); and a discussion of the causes and consequences of our self-inflicted stultification need to be repeated in this place. Instead, let me end with a few words concerning what the future may hold in store for us, and for our subject. That the onward march of human technology will not slow down - let alone come to a halt in our time - is obvious to any student of the subject at the present time - if anything, technology is the last manifestation 
of human activity likely to decline; and if so, where is astronomy likely to be in another two centuries - the time which separates us from the time when F. W. Bessel opened his young eyes in Minden on this old Universe of ours - now 200 years older?

With all refraction anomalies, or effects of thermal expansion of flexures of groundbased telescopes of Bessel's time now completely absent aboard the satellites operating in weightless conditions, how serious will be the effects of interplanetary or interstellar extinction on observations carried out beyond the confines of the terrestrial atmosphere; and how far shall we be able to see through interstellar haze in the extreme UV? How far will astronomy of double stars progress after observations of requisite precision will enable us to close the gap between 'wide' and 'close' binaries - which now increases with increasing distance from the Sun - and what all shall we yet learn about binaries with mass-ratios intermediate between those of stellar pairs and planetary systems? How many planetary systems exist in our neighbourhood, or in vaster domains of the Milky Way. In another 200 years we should know all this, and more - isn't it almost worthwhile to wait that long to see for ourselves?

\section{References}

Bender, P. L. et al. (12 co-authors): 1973, Science 182, 229.

Chapman, S.: 1918, Quart. J. Roy. Met. Soc. 44, 271.

Gorynia, A. A.: 1965, Nauch. Dumka (Ser. Astron. Astrophys), Kiev.

Habibullin, Sh. T.: 1966, Trudy Astron. Inst. Univ. Kazan, No. 34.

Jeffreys, H.: 1924, The Earth (first ed.), Cambridge Univ. Press, Cambridge.

Kopal, Z.: 1969, The Moon (second ed.), D. Reidel Publ. Co., Dordrecht, Holland, p. 88.

Kopal, Z.: 1974, The Moon in the Post-Apollo Era, D. Reidel Publ. Co., Dordrecht, Holland, p. 79.

Kopal, Z.: 1984, in C. Wickramasinghe (ed.), Fundamental Studies in the Future of Science, Cardiff Univ. Press, p. 231.

Koziel, K.: 1967a, Icarus 7, 1.

Koziel, K.: 1967b, in Z. Kopal and C. L. Goudas (eds.), Measure of the Moon, D. Reidel Publ. Co., Dordrecht, Holland, pp. 3-11.

Williams, J. G. et al. (4 authors): 1973, Moon 4, 190. 